\title{
RECOMMENDATIONS FROM A MEETING ON HEALTH IMPLICA- TIONS OF GENETICALLY MODIFIED ORGANISM (GMO)
}

\author{
Ghana Public Health Association
}

DOI: http://dx.doi.org/10.4314/gmj.v48i2.11

\section{EXECUTIVE SUMMARY}

The Ghana Public Health Association organized a scientific seminar to examine the introduction of genetically modified organisms into public use and the health consequences. The seminar was driven by current public debate on the subject. The seminar identified some of the advantages of GMOs and also the health concerns. It is clear that there is the need to enhance local capacity to research the introduction and use of GMOs; to put in place appropriate regulatory mechanisms including particularly the labeling of GMO products and post-marketing surveillance for possible negative health consequences in the long term. Furthermore the appropriate state agency should put in place advocacy strategies to keep the public informed about GMOs.

\section{CONTEXT}

There is universal agreement that the world's population is increasing at an alarming rate especially in developing countries and this poses a major threat to food security. The United Nations projects that world population will increase by $25 \%$ to 7.5 billion by 2020 . Climate change is likely to worsen available food supplies which may lead to malnutrition and other serious health problems.

To address these concerns, Genetically Modified Organisms (GMOs) have been introduced to improve food production. According to the EU, a Genetically Modified Organism is any organism, with the exception of human beings, in which the genetic material has been altered in a way that does not occur naturally by mating and/or natural recombination. Genetically Modified (GM) products include medicines and vaccines, foods and food ingredients, feeds, and fibers. As of August 2012, the United States of America Food and Drugs Administration has approved a total of 144 crops, including corn, soy, cotton, canola, potato, squash and tomato, for commercialization.

In Ghana, the Biosafety Act (2011), Act 831,was passed to establish the National Biosafety Authority with the objective of, inter alia, "to ensure an adequate level of protection in the field of safe development, transfer, handling and use of genetically modified organisms resulting from biotechnology that may have an adverse effect on health and the environment".
The law therefore allows the application of biotechnology in food crop production. The Act does not apply to "genetically modified organisms that are pharmaceutical for human use".

The Plant Breeders bill laid in Parliament on the 28th day of May, 2013 on the other hand seeks among others to provide for the grant and protection of plant breeder rights. This bill has generated such controversy that it has been withdrawn pending further consultation.

Biotechnological tools have been used to modify agricultural produce in Ghana's agricultural research institutes contributing to better yields, pest and weather resistance

There has been widespread controversy generated worldwide, Ghana included, since the introduction of GM crops, especially after commercialization of the products. Concerns have been raised about GM crops for a variety of reasons including Ethical, Biosafety (Environmental impact, Health), Bioproperty, and Biopolitics considerations.

It is in this context that the Ghana Public Health Association (GPHA) organized a public scientific lecture on GMOs and its health implications on $3^{\text {rd }}$ April 2014 to understand the science behind the technology and discuss its public health implications to inform policy. GPHA is an apolitical, non-governmental professional association of all interested professionals who have a contribution to make to improve public health in Ghana.

Experts from the College of Agriculture and Consumer Sciences of the University of Ghana, Legon, and National Public Health Surveillance Department of Ghana Health Service presented carefully researched scientific papers on the subject, followed by thorough dispassionate discussions. 


\section{SYNTHESIS OF ISSUES}

The presentations and discussions resulted in the elucidation of clear advantages and disadvantages of GMOs on health.

\section{Advantages:}

Some of the advantages and potential health benefits include the following:

- Introduction of pest resistant, herbicide tolerant, disease resistant, cold tolerant, and drought tolerant crops with potential for increased crop yield.

- Potential for reduced use of chemicals and pesticides on insect resistant plants, which will result in healthier food

- Improving the nutritional properties of crops to enhance human health through food fortification with desired vitamins and minerals

- GMOs provide huge potential for production of pharmaceuticals and vaccines which will be much easier to ship, store and administer than traditional injectable vaccines.

- Use of technology for Gene therapy - direct use of DNA to treat disease

- Application in stem cell research - the manipulation of undifferentiated cells to replace damaged or diseased tissues in the body

\section{CONCERNS}

Notwithstanding the advantages and major potential public health benefits, a number of concerns and potential negative health impact were identified:

- Current efforts are focused primarily on a few crop/trait combinations that have high commercial value and occupy large international markets, hence are primarily profit driven

- Public Institutions are resource limited and lack infrastructure and capacity to compete; there is poor access to advanced technology and weak regulatory capacity in country.

- $\quad$ Potential for unpredictable, unintended mutations in the organism with consequential medico-legal events.

- Tendency to provoke allergic reactions. New pesticide residue effects in alimentary system may affect gut bacteria provoking peripheral immune and allergic reactions.

- Lack of labeling of GMO products hinders postmarketing surveillance of the GMO products for safety.

- Most of the trials supporting introduction of GMOs are not independently done and are commercially driven. The trial designs are laboratory design and of short duration. Short-term studies may mask chronic toxicological effects including later developmental and reproductive effects.

- Increasing trends in organ disease and GMOs use have been observed. There is some correlation between organ disease, increasing GMO in food supply, and glyphosate herbal application with possibility of endocrine disruption. Organ disease mentioned include: Thyroid cancer; Liver and Intra hepatic bile duct cancer; Hypertension; Acute kidney disease; Diabetes mellitus; Autism; Alzheimer's disease; Parkinson's disease; Senile dementia; Inflammatory bowel disease - Crohn's and Ulcerative colitis; Rheumatoid arthritis. Though these have not been proven yet there is need to keep an eye on the correlation through further observational and epidemiological studies.

\section{CONCLUSIONS, RECOMMENDATIONS AND WAY FORWARD}

1. There is great potential to improve health, nutrition and food security through GMOs and Ghana should place itself appropriately to benefit maximally from the technology.

2. However, there is need to be cautious and move slowly. The development, testing and release of GMO products must be appropriately regulated. This calls for regulatory structures that ensure that there is no harm to humans, animals and the environment. The current systems in place are not adequate and capable to perform these critical functions.

3. Protocols for new GMO trials and release of GMO crops should be transparently reviewed by independent national regulatory body before approval.

4. The regulatory, clinical trial and post-marketing monitoring and surveillance capacities in the country should be strengthened. Universities and Research institutions should therefore be supported and equipped in the area of biotechnology.

5. There is need for longer, more-detailed transparent toxicological tests on all GMOs before approval and release to the market.

6. All GMOs must be appropriately labeled as such for informed choice and to facilitate post-market monitoring of any health effects

\section{POLICY OPTIONS/ DIRECTIONS}

1. The Biosafety Act (2011), Act 831, which has already been passed, can be maintained and an appropriate Legislative Instrument developed with wide consultations to take care of the health concerns and other conclusions and recommendations listed above.

2. Another option is to withdraw the Biosafety Act, if possible, for review to ensure that the recommen- 
dations and health concerns are taken care of in a new parent Biosafety Act.

3. Another option is to incorporate the health concerns and recommendations in the proposed Plant Breeders bill as appropriate.
Dr George Amofah MB. ChB, MPH, FGCP General Secretary

Ghana Public Health Association

E-mail: george.amofah@ghsmail.org 